\title{
Ligation assisted endoscopic mucosal resection of neuroendocrine tumor of duodenal bulb: a case report with a follow up of 24 months
}

\author{
Umid Kumar Shrestha, Gopi Aryal \\ Nepal Mediciti Hospital, Bhaisepati, Lalitpur, Nepal
}

Keywords: Duodenal neuroendocrine tumor; endoscopic mucosal resection; band ligation technique

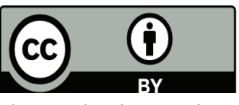

This work is licensed under a Creative Commons Attribution 4.0 Unported License.

\section{Introduction}

Duodenal neuroendocrine tumor (NET) is a rare solitary lesion arising from the mucosa and submucosa of the duodenum, which is found incidentally during upper gastrointestinal endoscopy. The tumor originates from the enterochromaffin cells of the gastroenteropancreatic neuroendocrine system. The primary duodenal NETs grow slowly and show an indolent clinical behavior, but they are potentially malignant. ${ }^{1}$ The duodenal NET without malignancy features may be treated optimally by the endoscopic resection. ${ }^{2}$ Eendoscopic Mucosal Resection (EMR) has been the commonly used endoscopic procedure for duodenal carcinoid tumors. It was seen that the higher complete resection rate for rectal or esophageal submucosa tumors was achieved with ligation-assisted EMR than conventional EMR., ${ }^{3,4}$ The EMR procedure itself is simple, safe, and time-saving, but EMR done to resect duodenal NETs is likely to have positive vertical margins. ${ }^{5}$ The Endoscopic Submucosal Dissection (ESD) may yield a higher

\begin{abstract}
Duodenal neuroendocrine tumor (NET) is a rare solitary lesion arising from the mucosa and submucosa of the duodenum, which is found incidentally during upper gastrointestinal endoscopy. Eendoscopic Mucosal Resection (EMR) has been the commonly used endoscopic procedure for duodenal carcinoid tumors, but the conventional EMR done to resect duodenal NETs is likely to have positive vertical margins. However, the ligation assisted EMR has recently been shown to be a promising technique for the treatment of duodenal NET that can have a negative free margin. In our study, we present a patient of 51-year-old male, who presented with pain over epigastrium and upper gastrointestinal endoscopy revealed a small submucosal lesion of $10 \mathrm{~mm}$ in the duodenal bulb. The endoscopic ultrasound showed the lesion arising from the echo layer three. The biopsy was taken which showed the duodenal NET. The computed tomography of abdomen did not show any evidence of distant metastasis. The EMR of duodenal NET was done by band ligation technique. The biopsy from the resected duodenal lesion confirmed the duodenal NET with the margin free of the tumor. The patient was followed at 6 , 12,18 and 24 months. During the follow up visits, the repeat upper gastrointestinal endoscopy did not show recurrence of the lesion and there was no any evidence of distant metastasis either. Ligation assisted EMR is an acceptable treatment in the hands of expert for small duodenal NET without the evidence of metastasis and can ensure the complete removal of the lesion with vertical free margin.
\end{abstract}

Correspondence Author:

Umid Kumar Shrestha, MD, PhD, FACG

Head, Department of Gastroenterology, Hepatology and Endoscopy Nepal Mediciti Hospital, Bhaisepati, Lalitpur, Nepal

Email: umidshrestha@gmail.com 
margin-free resection rate than EMR, but there is a higher risk of perforation with ESD, when performed by endoscopists with less experience or skill in endoscopy. ${ }^{6}$ However, the ligation assisted EMR has recently been shown to be a promising technique for the treatment of duodenal NET that can have a negative free margin. Recent study done in 10 patients showed that if the duodenal NET is located in the submucosal layer, is less than $13 \mathrm{~mm}$ in diameter, and is free of metastasis, ligation assisted EMR can successfully remove duodenal NET with a negative vertical margin. ${ }^{5}$

\section{Case Description}

In our study, we present a patient of 51-year-old male, who presented with pain over epigastrium and upper gastrointestinal endoscopy revealed a small submucosal lesion of $10 \mathrm{~mm}$ with a broad base in the duodenal bulb (figure 1). The endoscopic ultrasound showed the lesion arising from the echo layer three. The biopsy was taken which showed the duodenal NET. The computed tomography of abdomen did not show any evidence of distant metastasis.

The EMR of duodenal NET was done by band ligation technique. During the procedure, the submucosal injection of $10 \mathrm{ml}$ of Normal Saline along with Adrenaline $(1: 20,000)$ and Methylene Blue was given to raise the lesion, which was then grabbed with the band ligation, followed by its resection with the snare (figure 2 and 3). The hemoclips were applied in the mucosal defect area of the lesion. There were no significant bleeding, no perforation and no any other complications.

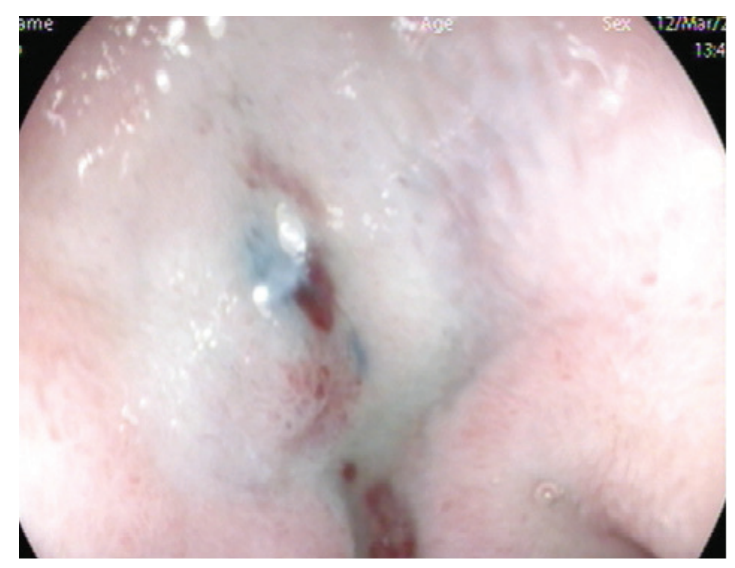

Figure 1: Upper Gastrointestinal endoscopy showed small submucosal lesion of $10 \mathrm{~mm}$ with a broad base in the duodenal bulb

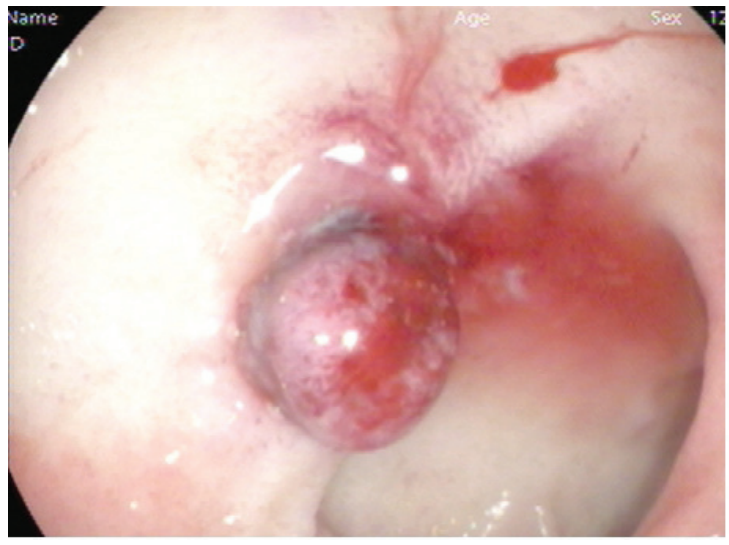

Figure 2: Duodenal lesion was raised with saline submucosal injection and was grabbed with band ligation

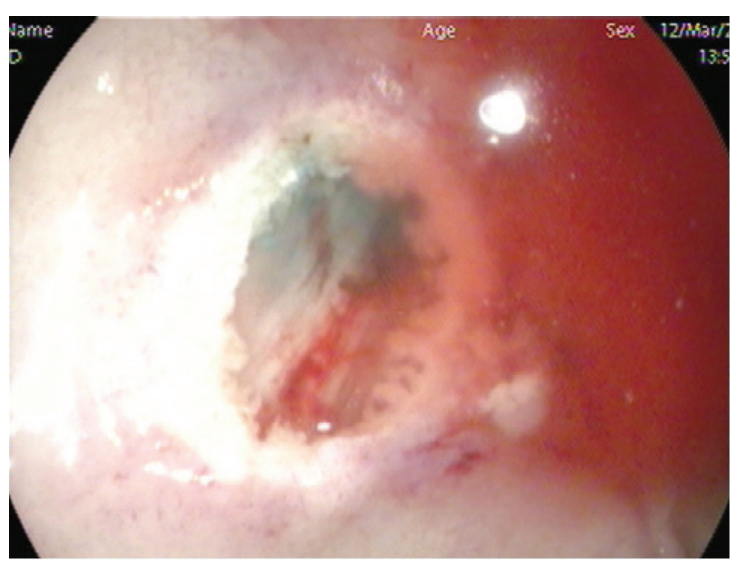

Figure 3: Duodenal submucosal lesion was resected endoscopically with a snare

The biopsy from the resected duodenal lesion confirmed the duodenal NET with the margin free of the tumor. The patient was followed at 6, 12, 18 and 24 months. During the follow up visits, the repeat upper gastrointestinal endoscopy did not show recurrence of the lesion and there was no any evidence of distant metastasis either.

\section{Discussion}

HDuodenal NETs without metastasis may be treated by conventional surgical operations, but it may be unduly invasive and determining the appropriate extent of resection for duodenal lesions may be difficult. ${ }^{7}$ As far as possible, the possible treatment for duodenal NET without metastasis should be less-invasive endoscopic resection, as it provides an improved quality of life compared to surgical resection. The European Neuroendocrine Tumor Society has recommended that duodenal NET, with size of $<10 \mathrm{~mm}$ in diameter, limited to the submucosal layer, and without lymph node involvement or distant metastasis, be treated endoscopically. ${ }^{8}$

However, the choice of method of endoscopic treatment for duodenal NET without metastasis has been controversial. The ESD may be the acceptable option for duodenal NET, but the 
procedure is technically difficult and the risk of perforation is high because the duodenal wall is generally thinner than the wall of the stomach. ${ }^{9}$ The EMR is another option for the treatment of duodenal NET, but the likelihood of positive vertical margin is high with the conventional EMR. ${ }^{10}$ In the present study, we used band ligation technique of EMR to resect the duodenal NET, which was $10 \mathrm{~mm}$ in size and was free of observable metastasis. We used normal saline and methylene blue to lift the submcuosa and to color the lesion, respectively. We did an en bloc resection of a duodenal NET with negative vertical margin by snaring under the band.

\section{Conclusion}

Ligation assisted EMR is an acceptable treatment in the hands of expert for small duodenal NET without the evidence of metastasis and can ensure the complete removal of the lesion with vertical free margin.

\section{References}

1. Kim SH,Park $\mathrm{CH}, \mathrm{Ki} \mathrm{HS}$, et al. Endoscopic Treatment of Duodenal Neuroendocrine Tumors. Clin Endosc 2013;46:656-661

2. Park SB, Kang DH, Choi CW, et al. Clinical outcomes of ligationassisted endoscopic resection for duodenal neuroendocrine tumors. Medicine 2018; 97:18(e0533)

3. Choi CW, Kang DH, Kim HW, et al. Comparison of endoscopic resection therapies for rectal carcinoid tumor: endoscopic submucosal dissection versus endoscopic mucosal resection using band ligation. J Clin Gastroenterol 2013;47:432-6

4. Choi CW, Kang DH, Kim HW, et al. Endoscopic resection for small esophageal submucosa tumor: band ligation versus conventional endoscopic mucosal resection. Medicine (Baltimore) 2017;96:e7574.

5. Fujimoto A, Sasaki M, Goto O, et al. Treatment Results of Endoscopic Mucosal Resection with a Ligation Device for Duodenal Neuroendocrine Tumors. Intern Med 58: 773-777, 2019

6. Suzuki S, Ishii N, Uemura M, et al. Endoscopic submucosal dissection (ESD) for gastrointestinal carcinoid tumors. Surg Endosc 26: 759-763, 2012.

7. Iwasaki $T$, Nara $S$, Kishi $Y$, et al. Surgical treatment of neuroendocrine tumors in the second portion of the duodenum: a single center experience and systematic review of the literature. Langenbecks Arch Surg 402: 925-933, 2017

8. Klöppel G, Couvelard A, Perren A, et al. ENETS Consensus Guidelines for the Standards of Care in Neuroendocrine Tumors: towards a standardized approach to the diagnosis of gastroenteropancreatic neuroendocrine tumors and their prognostic stratification. Neuroendocrinology 90: 162-166, 2009.

9. Suzuki S, Ishii N, Uemura M, et al. Endoscopic submucosal dissection (ESD) for gastrointestinal carcinoid tumors. Surg Endosc 26: 759-763, 2012.

10. Kim GH, Kim Jl, Jeon SW, et al.; Korean College of Helicobacter and Upper Gastrointestinal Research. Endoscopic resection for duodenal carcinoid tumors: a multicenter, retrospective study. J Gastroenterol Hepatol 29: 318-324, 2014. 